Pacific Journal of Mathematic 


\title{
THE STRATIFICATION OF COMPACT CONNECTED LIE GROUP ACTIONS BY SUBTORI
}

\author{
Christopher Allday
}

\begin{abstract}
A stratification of the spectrum of the $\bmod p$ equivariant cohomology ring of a compact Lie group action in terms of elementary $p$-subgroups has been obtained by Quillen. A corresponding result for compact connected Lie group actions in terms of subtori is proven in this paper by different means. In addition some localization and primary decomposition theorems are obtained. The paper closes with an application to uniform torus actions.
\end{abstract}

Introduction. In [11] Quillen proves a stratification theorem for compact Lie group actions by elementary abelian $p$-subgroups in equivariant cohomology with coefficients in $\mathbf{Z} / p \mathbf{Z}$. In the first section of this paper we prove the stratification theorem for compact connected Lie group actions by subtori in equivariant cohomology with rational coefficients. We do not attempt to follow Quillen's method of proof, however. There are three reasons for this. First, the existence in Quillen's situation of a "universal invertible" (the element $\boldsymbol{e}_{\mathrm{A}}$ of Theorem 4.2 of [10]) has no natural analogue in our situation. Secondly, Quillen's vital Main Theorem (Theorem 6.2 of [10]) appears to require certain restrictions on the orbit structure which can be avoided. (See Lemma 1.5, below). Thirdly, the results and techniques of Hsiang, Chang and Skjelbred ([7], [6] and [13]) give rise to a proof which is more direct and less sophisticated than the proof Quillen gives for his theorem.

In the second section of this paper, we prove a localization theorem for $H_{T}(X)$-module structures and deduce analogues of the results of [6], using $H_{T}(X)$-module structures instead of $H\left(B_{T}\right)$-module structures. The advantage of this approach is that it distinguishes between components of the fixed point set of a subtorus. In the third section, by way of an application, the useful concept of a uniform torus action is defined, and a simple algebraic characterization of uniformity is given.

Throughout this paper the cohomology and equivariant cohomology theory used will be sheaf theoretic, or equivalently, Čech. Rational coefficients will be used throughout, and these will be suppressed from the notation. The form of the "going up" theorem of Cohen and Seidenberg, which is used in the first section, is that which may be found in Serre ([12]).

We wish to thank Professor W.-Y. Hsiang, T. Chang and T. Skjelbred for many useful discussions and suggestions. 
1. The stratification theorem. Let $G$ be a compact connected Lie group, and let $X$ be a topological space, on which $G$ acts continuously. Throughout this paper we shall assume that the $G$-space $X$ satisfies one of the following two assumptions:

(A) $X$ is compact (Hausdorff), and $\operatorname{dim}_{\mathrm{Q}} H^{*}(X)<\infty$;

(B) $X$ is paracompact, $c d_{\mathbf{Q}}(X)<\infty$, $\operatorname{dim}_{\mathbf{Q}} H^{*}(X)<\infty$, and $G$ acts on $X$ with finitely many orbit types.

We shall be concerned with the set $\mathscr{T}(X)$ of all pairs $(K, c)$, where $K$ is a subtorus of $G$, such that $X^{K} \neq \varnothing$, and $c$ is a component of $X^{K}$. $\quad \mathscr{T}(X)$ will be partially ordered as follows:

for $(H, d)$ and $(K, c)$ in $\mathscr{T}(X),(H, d) \leqq(K, c)$, if and only if there exists $g \in G$, such that $g H g^{-1} \subseteq K$ and $g d \supseteq c$.

If $(H, d) \leqq(K, c)$ and $(K, c) \leqq(H, d)$, then we shall write $(H, d) \equiv(K, c)$. Thus, in $\mathscr{T}(X),(H, d) \equiv(K, c)$ if and only if there exists $g \in G$, such that $g H g^{-1}=K$ and $g d=c$.

Following Quillen [11], let $H_{G}(X)=H_{G}^{\text {even }}(X)$, and let $H_{L}=H^{*}\left(B_{L}\right)$, for any closed subgroup $L \subseteq G$. A pair $(K, c) \in \mathscr{T}(X)$ determines a cross-section of the bundle $X_{K} \rightarrow B_{K}$, and hence a map $B_{K} \rightarrow X_{G}$. The induced map on cohomology, $H_{G}(X) \rightarrow H_{K}$, will be denoted by $(K, c)^{*}$, and the kernel of $(K, c)^{*}$ will be denoted by $\mathbf{p}(K, c)$. Since we are using sheaf-theoretic cohomology, $(K, c)^{*}$ is independent of the choice of point in $c$ used to determine the cross-section $B_{K} \rightarrow X_{K}$. It is clear, too, that if $(H, d) \equiv(K, c)$ in $\mathscr{T}(X)$, then $\mathbf{p}(H, d)=\mathbf{p}(K, c)$.

Continuing to recall the notation of [11], let

$$
V(K, c)=\left\{\mathbf{p} \in \operatorname{Spec}\left(H_{G}(X)\right) \mid \mathbf{p} \supseteq \mathbf{p}(K, c)\right\},
$$

and let $V(K, c)^{+}=V(K, c)-U\{V(H, d) \mid \mathbf{p}(H, d) \supset \mathbf{p}(K, c)\}$.

Let $\operatorname{Norm}(K, c)=\left\{g \in G \mid g K g^{-1}=K\right.$ and $\left.g c=c\right\}$, and let $Z K=$ $\left\{g \in G \mid g x g^{-1}=x\right.$, for all $\left.x \in K\right\}$. Since the centralizer of $K, Z K$, is connected (see, for example, Bredon [5]), $g c=c$, for all $g \in Z K$, and all components $c$ of $X^{K}$. Let $T$ be any maximal torus in $G$, and let $N T$ be the normalizer of $T$ in $G$. Thus the Weyl group of $G, W$, is equal to $N T / T$. If $K \subseteq T$, let $W(K, c)$ be the group $\operatorname{Norm}(K, c) \cap N T / Z K \cap$ NT.

Given a maximal torus $T \subseteq G$, and given $(H, d) \in \mathscr{T}(X)$, there exists $(K, c) \in \mathscr{T}(X)$ such that $K \subseteq T$ and $(K, c) \equiv(H, d)$. Thus we may find a subset $I_{T}$ of $\mathscr{T}(X)$, such that

(i) $(K, c) \in I_{T} \Rightarrow K \subseteq T$, and

(ii) for any $(H, d) \in \mathscr{T}(X)$, there exists one and only one $(K, c) \in I_{T}$ such that $(H, d) \equiv(K, c)$. 
Recall from Chang and Skjelbred [6] that for a torus, $K$, there is a function

$$
\sigma: \operatorname{Spec}\left(H_{K}\right) \rightarrow\left\{P L \in \operatorname{Spec}\left(H_{K}\right) \mid L \text { is a subtorus of } K\right\},
$$

which assigns to each prime ideal $\mathbf{p}$ of $H_{K}$, the ideal generated by $\mathbf{p} \cap H^{2}\left(B_{K}\right)$. Thus $\mathbf{p} \supseteq P L$ if and only if $\sigma(\mathbf{p}) \supseteq P L$. $\sigma(\mathbf{p})$ will be called the support of $\mathbf{p}$. Let $K^{+}=\left\{\mathbf{p} \in \operatorname{Spec}\left(H_{K}\right) \mid \sigma(\mathbf{p})=(0)\right\}$.

The remainder of this section will be devoted to proving the following version of the Stratification Theorem.

THEOREM 1.1. Let $T$ be a maximal torus of $G$. Then

(i) The spectrum of $H_{G}(X)$ admits a stratification as a disjoint union

$$
\operatorname{Spec}\left(H_{G}(X)\right)=\coprod_{(K, c) \in I_{T}} V(K, c)^{+}
$$

(ii) $W(K, c)$ acts on $K^{+}$, and there is a homeomorphism

$$
K^{+} / W(K, c) \rightarrow V(K, c)^{+}
$$

(iii) for any $(H, d)$ and $(K, c)$ in $\mathscr{T}(X), \mathbf{p}(H, d) \supseteq \mathbf{p}(K, c)$ if and only if $(H, d) \leqq(K, c)$.

REMARKS 1.2 .

(i) Theorem 1.1 (i) is equivalent to asserting the existence of a function

$$
\sigma: \operatorname{Spec}\left(H_{G}(X)\right) \rightarrow\left\{\mathbf{p}(K, c) \mid(K, c) \in I_{T}\right\}
$$

with the property that, for any $(H, d) \in \mathscr{T}(X), \mathbf{p} \supseteq \mathbf{p}(H, d)$ if and only if $\sigma(\mathbf{p}) \supseteq \mathbf{p}(H, d) . \quad \sigma(\mathbf{p})$ will be called the support of $\mathbf{p}$.

(ii) In the above remark we have used the fact that the commutative ring $H_{G}(X)$ is Noetherian. This is clear since $H_{G}$ is Noetherian, and the fact that $\operatorname{dim}_{Q} H^{*}(X)<\infty$ implies that $H_{G}(X)$ is a finitely generated $H_{G}$-algebra ([10]).

(iii) The sufficiency of the condition in Theorem 1.1 (iii) is clear, since $(H, d) \leqq(K, c)$ implies that we may factorize $(H, d)^{*}$ through $(K, c)^{*}$.

(iv) If $S_{0}$ is the multiplicative set generated by nonzero elements in $H^{2}\left(B_{K}\right)$, then $K^{+}$is homeomorphic to $\operatorname{Spec}\left(S_{0}^{-1} H_{K}\right)$.

(v) All topologies on ring spectra used above are Zariski topologies. 
(vi) $W(K, c)$ has the discrete topology. Since we have an epimorphism $[\operatorname{Norm}(K, c) \cap N T] / T \rightarrow W(K, c)$, and a monomorphism $[\operatorname{Norm}(K, c) \cap N T] / T \rightarrow W$, it is clear that $W(K, c)$ is finite, with order less than or equal to the order of $W$.

The following proposition will enable us to prove Theorem 1.1 by two separate applications of the going up theorem of Cohen and Seidenberg. $\quad \pi$ is the bundle map $X_{T} \rightarrow B_{T}, R=H_{T}$, and $J$ is the kernel of $\pi^{*}: R \rightarrow H_{T}(X) . \quad \pi^{*}$ will be denoted by $\rho$. Part (ii) is due to T. Skjelbred [8]: we include his proof.

Proposition 1.3.

(i) $H_{T}(X)$ is an integral extension of $R / J$.

(ii) $H_{T}(X)$ is a finite Galois extension of $H_{G}(X)$. In particular the extension is integral.

Proof.

(i) Let $x \in H_{T}(X)$. Then the $R$-submodule of $H_{T}(X)$ generated by powers of $x$ is finitely generated.

(ii) Skjelbred has shown that it is an easy consequence of a theorem of Borel, that the natural map $H_{G}(X) \rightarrow H_{T}(X)$ is an isomorphism of $H_{G}(X)$ onto $H_{T}(X)^{W}$, the subring of $H_{T}(X)$ fixed by the natural action of $W([8])$. For $x \in H_{T}(X)$, consideration of $\prod_{w \in W}(x-w x)$ completes the proof.

REMARK. In [7] W.-Y. Hsiang shows that $\sqrt{J}=\bigcap_{\imath=1}^{m} P K_{\imath}$, where $K_{1}, \cdots, K_{m}$ are the maximal connective isotropy subgroups of the action of $T$ on $X$.

For the time being we shall assume that $G=T$, a torus, and we shall prove Theorem 1.1 in this case. For a subtorus $K \subseteq T$, let $\beta_{K}$ be the map $X_{K} \rightarrow X_{T}$, and let $Q K$ be the ideal generated by $\rho(P K)$ in $H_{T}(X)$. If $K_{x}^{\circ}$ is the identity component of the isotropy subgroup of the action of $K$ on $X$ at $x \in X$, let $F(K, x)$ be the component of $X^{K_{k}^{\circ}}$, which includes $x$.

Proposition 1.4. In $H_{T}(X)$,

$$
\sqrt{\operatorname{ker}\left(\beta_{K}^{*}\right)}=\sqrt{Q K}=\bigcap_{x \in X} \mathbf{p}\left(K_{x}^{\circ}, F(K, x)\right) .
$$

Proof. To prove that $\sqrt{\operatorname{ker}\left(\beta_{K}^{*}\right)}=\sqrt{Q K}$, consider the Serre spectral sequence in rational cohomology of the fibration $X_{K} \stackrel{\beta_{K}}{\rightarrow} X_{T} \stackrel{q}{\rightarrow} B_{L}$, where $L=T / K . R=H_{L} \otimes H_{K}$ acts on $E_{2}$ via the action of $H_{L}$ on itself, 
and the action of $H_{K}$ on $H^{*}\left(X_{K}\right) . \quad E_{2}$ is a finitely generated bigraded differential $R$-module.

Since $E_{x}$ is a subquotient of $E_{2}$ and $R$ is Noetherian, it follows that $E_{x}=E_{r}$ for some $r<\infty$, and $E_{r}$ is a finitely generated $R$-module. Thus, with the standard filtration, there exists a set of generators for $E_{r}$ as an $H_{L}$-module having bounded filtration degree. Let $n$ be an integer greater than the maximum filtration degree of the elements of such a generating set. Let $x \in \operatorname{ker} \beta_{K}^{*}$. Then $x \in F_{1} H_{T}^{*}(X)$, and so $x^{n} \in$ $F_{n} H_{T}^{*}(X)$.

It now follows that there exist $y_{1}, \cdots, y_{m}$ in $H_{T}^{*}(X)$, and $a_{1}, \cdots, a_{m}$ of positive degree in $H_{L}$, such that $x^{n}=a_{1} y_{1}+\cdots+a_{m} y_{m}$. But $q^{*}$ maps the elements of positive degree in $H_{L}$ into $\rho(P K)$. Hence, if $x \in$ $\operatorname{ker}\left(\beta_{K}^{*} \mid H_{T}(X)\right)$, then $x^{n} \in Q K$.

Clearly, $Q K \subseteq \operatorname{ker}\left(\beta_{K}^{*}\right)$.

We must prove now that $\sqrt{\operatorname{ker}\left(\beta_{K}^{*}\right)}=\bigcap_{x \in X} \mathbf{p}\left(K_{x}^{\circ}, F(K, x)\right)$. Given a subtorus $L \subseteq K$, and a component $c$ of $X^{L}$, let $(L, c)^{\prime *}: H_{K}(X) \rightarrow H_{L}$ be the associated map, and let $\mathbf{p}^{\prime}(L, c)=\operatorname{ker}(L, c)^{\prime *}$. Then $\mathbf{p}(L, c)=$ $\beta_{K}^{*-1}\left(\mathbf{p}^{\prime}(L, c)\right)$.

Now Quillen's Proposition 3.2 of [10] implies that in $H_{K}(X)$, $\sqrt{(0)} \equiv \bigcap_{x \in X} \mathbf{p}^{\prime}\left(K_{x}^{\circ}, F(K, x)\right)$. Hence

$$
\sqrt{\operatorname{ker}\left(\beta_{K}^{*}\right)}=\beta_{K}^{*-1}(\sqrt{(0)})=\bigcap_{x \in X} \mathbf{p}\left(K_{x}^{\circ}, F(K, x)\right) .
$$

The next lemma enables us to dispense with any condition on the number of orbit types when $X$ satisfies condition (A).

LEMMA 1.5. If a torus $K$ acts on a space $X$ satisfying condition (A), then the family

$$
\left\{\mathbf{p}\left(K_{x}^{\circ}, F(K, x)\right) \mid x \in X\right\} \subseteq \operatorname{Spec}\left(H_{K}(X)\right)
$$

has only finitely many minimal members.

Proof. By Remarks 1.2 (iii) it is enough to show that the family $F=\left\{\left(K_{x}^{\circ}, F(K, x)\right) \mid x \in X\right\}$ has only finitely many maximal members. Let $0 \leqq r \leqq \operatorname{rank}(K)$, and let $F_{r}=\left\{\left(K_{x}^{\circ}, F(K, x)\right) \mid x \in X\right.$ and corank $\left.\left(K_{x}^{\circ}\right)=r\right\}$. Let $S_{r-1}=\left\{x \in X \mid \operatorname{corank}\left(K_{x}^{\circ}\right) \leqq r-1\right\}$.

If $\left(K_{x}^{\circ}, F(K, x)\right)$ is in $F_{r}$ and is maximal in $F$, then $F(K, x) \cap S_{r-1}=\varnothing$. Hence, by the Localization Theorem ([6]), $P K_{x}^{\circ} \in \operatorname{Supp}\left(H_{K}^{*}\left(X, S_{r-1}\right)\right)$. Furthermore, $P K_{x}^{\circ}$ is clearly minimal in $\operatorname{Supp}\left(H_{K}^{*}\left(X, S_{r-1}\right)\right)$. But, from [2], $\left.H_{K}^{*}\left(X, S_{r-1}\right)\right)$ is a finitely generated $H_{K}$-module, and so $\operatorname{Supp}\left(H_{K}^{*}\left(X, S_{r-1}\right)\right)$ has only finitely many minimal elements. 
REMARKS.

(1) From the commuting diagram

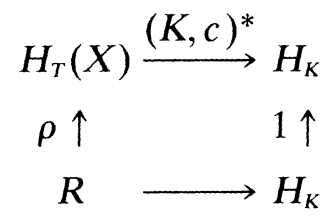

it follows that $\rho^{-1}(\mathbf{p}(K, c))=P K$.

(2) Since $\sqrt{(0)}=\bigcap_{x \in X} \mathbf{p}\left(T_{x}^{\circ}, F(T, x)\right)$, every prime ideal in $H_{T}(X)$ contains some $\mathbf{p}(K, c)$.

To establish the existence of supports we need the following lemma:

Lemma 1.6. Let $\mathbf{p} \in \operatorname{Spec}\left(H_{T}(X)\right)$. If $\rho^{-1}(\mathbf{p})=P K$, then there is a component $c$ of $X^{K}$ such that $\mathbf{p}=\mathbf{p}(K, c)$.

Proof. $\rho^{-1}(\mathbf{p})=P K \Rightarrow \mathbf{p} \supseteq Q K$. Hence by Proposition 1.4 (and Lemma 1.5), there exists $x \in X$ such that $\mathbf{p} \supseteq \mathbf{p}\left(K_{x}^{\circ}, F(K, x)\right)$.

Therefore, $P K=\rho^{-1}(\mathbf{p}) \supseteq \rho^{-1}\left(\mathbf{p}\left(K_{x}^{\circ}, F(K, x)\right)=P K_{x}^{\circ}\right.$. But $K_{x}^{\circ} \subseteq K$, and so $P K_{x}^{\circ} \supseteq P K$. Hence $K=K_{x}^{\circ}$, and $\mathbf{p}$ and $\mathbf{p}(K, c)$, where $c=$ $F(K, x)$, are two prime ideals of $H_{T}(X)$ lying over the prime ideal $\rho(P K)$ in $R / J$, with $\mathbf{p} \supseteq \mathbf{p}(K, c)$. The result follows by the Cohen-Seidenberg Theorem from Proposition 1.3 (i). (Note that $P K \supseteq J$, since $K$ is contained in $T_{x}^{\circ}$ ).

The next lemma is straightforward.

Lemma 1.7. For any $(K, c)$ in $\mathscr{T}(X)$, the map

$$
r+P K \rightarrow \rho(r)+\mathbf{p}(K, c)
$$

is an isomorphism $\psi: R / P K=H_{K} \rightarrow H_{T}(X) / \mathbf{p}(K, c)$.

Lemma 1.8. Let $\mathbf{p} \in \operatorname{Spec}\left(H_{T}(X)\right)$, let $P L=\sigma \rho^{-1}(\mathbf{p})$, and let $d$ and $d^{\prime}$ be components of $X^{L}$. If $\mathbf{p} \supseteq \mathbf{p}(L, d)+\mathbf{p}\left(L, d^{\prime}\right)$, then $d=d^{\prime}$.

Proof. Suppose $d \neq d^{\prime}$, and let $d=d_{1}, d^{\prime}=d_{2}, \cdots, d_{s}$ be the components of $X^{L}$. Let $x$ be the element $(1,0, \cdots, 0)$ in $H_{T}^{\circ}\left(X^{L}\right)=$ $H_{T}^{\circ}\left(d_{1}\right) \oplus \cdots \oplus H_{T}^{\circ}\left(d_{S}\right)$. Following the notation and methods of [6], let

$$
I_{x}=\left\{a \in R \mid a x \in \operatorname{Im}\left[\varphi_{L}^{*}: H_{T}^{*}(X) \rightarrow H_{T}^{*}\left(X^{L}\right)\right]\right\} .
$$

Let $a \in I_{x}$. Then $\varphi_{L}^{*} \rho(a)=a \cdot(1,1, \cdots, 1)$. There exists $y \in$ $H_{T}(X)$, such that $\varphi_{L}^{*}(y)=a x=a \cdot(1,0, \cdots, 0)$. Hence $y \in \mathbf{p}\left(L, d^{\prime}\right)$, and $\rho(a)-y \in \mathbf{p}(L, d)$. Thus $\rho a \in \mathbf{p}$. 
We have, then, that $\rho^{-1}(\mathbf{p}) \supseteq I_{x}$, and hence, $P L=\sigma \rho^{-1}(\mathbf{p})$ contains some $P H$, where $H$ belongs to $x$. Since it is clear that the subtori, which belong to $x$, cannot contain $L$, we have a contradiction.

If $d$ and $d^{\prime}$ are distinct components of $X^{L}$, for $L$ a subtorus of $T$, we shall say that a pair $(K, c)$ in $\mathscr{T}(X)$ connects $d$ and $d^{\prime}$ if $K \subseteq L$ and $d \cup d^{\prime} \subseteq c$. Then the following corollary is deduced easily from the above.

COROllary 1.9. $\sqrt{\mathbf{p}(L, d)+\mathbf{p}\left(L, d^{\prime}\right)}=\bigcap_{i=1}^{r} \mathbf{p}\left(K_{t}, c_{1}\right)$, where $\left(K_{1}, c_{1}\right)$, $\cdots,\left(K_{r}, c_{r}\right)$ are the maximal pairs of $\mathscr{T}(X)$, which connect $d$ and $d^{\prime}$.

Lemma 1.10. Let $A$ be a $Q$-linear subspace of $H_{T}(X)$, such that $\mathbf{p}(K, c) \subseteq A . \quad$ Then

$$
A=\mathbf{p}(K, c)+(A \cap \rho(R)),
$$

as $Q$-linear subspaces.

Thus, if $A$ is an ideal in $H_{T}(X)$, and $A^{\prime}$ is the ideal generated by $A \cap \rho(R)$, then $A=\mathbf{p}(K, c)+A^{\prime}$, as ideals.

In particular, if $(K, c) \geqq(L, d)$, then

$$
\mathbf{p}(L, d)=\mathbf{p}(K, c)+Q L .
$$

Proof. Clearly $\mathbf{p}(K, c)+(A \cap \rho(R)) \subseteq A$. Let $a \in A$, and let $q: R \rightarrow R / P K, \quad \bar{q}: H_{T}(X) \rightarrow H_{T}(X) / \mathbf{p}(K, c)$ be the projections. By Lemma 1.7, there exists $r \in R$ such that $\bar{q} \rho(r)=\psi q(r)=\bar{q}(a)$. Thus $\rho(r)-a \in \operatorname{ker} \bar{q}=\mathbf{p}(K, c) \subseteq A$, and so $\rho(r) \in A \cap \rho(R)$. Hence $a \in$ $\mathbf{p}(K, c)+(A \cap \rho(R))$.

We shall now prove Theorem 1.1 (iii) for a torus acting on $X$.

Lemma 1.11. If $\mathbf{p}(H, d) \supseteq \mathbf{p}(K, c)$, then $(H, d) \leqq(K, c)$.

Proof. By applying $\rho^{-1}$ we have that $P H \supseteq P K$, and hence $H \subseteq$ $K$. Thus there exists a component $d^{\prime}$ of $X^{H}$, such that $d^{\prime} \supseteq c$; and so $\mathbf{p}\left(H, d^{\prime}\right) \supseteq \mathbf{p}(K, c)$ also. Lemma 1.10 implies that

$$
\mathbf{p}(H, d)=\mathbf{p}\left(H, d^{\prime}\right)=\mathbf{p}(K, c)+Q H .
$$

It follows that $\mathbf{p}(H, d)=\mathbf{p}(H, d)+\mathbf{p}\left(H, d^{\prime}\right)$, and so $d^{\prime}=d$, by Lemma 1.8 .

The next lemma, together with Lemma 1.8 completes the proof of Theorem 1.1 (i) for torus actions.

Lemma 1.12. If $\mathbf{p} \supseteq \mathbf{p}(K, c)$ in $H_{T}(X)$, and if $P L=\sigma \rho^{-1}(\mathbf{p})$, then there exists a component $d$ of $X^{L}$, such that 


$$
\mathbf{p} \supseteq \mathbf{p}(L, d) \supseteq \mathbf{p}(K, c) \text {. }
$$

Proof. By Lemma 1.10,

$$
\mathbf{p}=\mathbf{p}(K, c)+\rho \rho^{-1}(\mathbf{p}) \supseteq \mathbf{p}(K, c)+\rho(P L)=\mathbf{p}(L, d),
$$

where $d$ is the component of $X^{L}$, which contains $c$.

Suppose that $X$ is a compact rational Poincaré duality space with $X^{T} \neq \varnothing$. Let $F$ be a component of $X^{T}$, and let $f \in H_{T}^{*}\left(X^{T}\right)$ be the top class of $F$. Let

$$
I_{f}=\left\{a \in R \mid a f \in \operatorname{Im}\left[\varphi^{*}: H_{T}^{*}(X) \rightarrow H_{T}^{*}\left(X^{T}\right)\right]\right\}
$$

Let $I_{f}^{\prime}$ be the ideal generated by $I_{f}$ in $H_{T}(X)$; and let $J_{f}$ be the annihilator in $H_{T}(X)$ of the $H_{T}(X)$-submodule of $H_{T}^{*}\left(X, X^{T}\right)$ generated by $\delta f$. Then $J_{f}=\left\{x \in H_{T}(X) \mid \varphi^{*}(x) f \in \operatorname{Im} \varphi^{*}\right\}$, and we have the following corollary of Lemma 1.10 .

Corollary 1.13. $J_{f}=I_{f}^{\prime}+\mathbf{p}(T, F)$, and $\sqrt{J_{f}}=\bigcap_{i=1}^{r} \mathbf{p}\left(K_{i}, c_{i}\right)$, where $K_{1}, \cdots, K_{r}$ are the local weights at $F$, with corresponding $F$-varieties $c_{1}, \cdots, c_{r}$, respectively.

Proof. Clearly $J_{f} \supseteq \mathbf{p}(T, F)$, and $J_{f} \cap \rho(R)=\rho\left(I_{f}\right)$. Thus $J_{f}=$ $I_{f}^{\prime}+\mathbf{p}(T, F)$ by Lemma 1.10 .

For the second part, we have that $\sqrt{I}_{f}=\bigcap_{t=1}^{r} P K_{l}$, by [1]; and, letting $c_{t}$ be the component of $X^{K_{i}}$, which contains $F$, we have, by Lemma 1.10 again,

$$
\begin{aligned}
\bigcap_{i=1}^{r} \mathbf{p}\left(K_{i}, C_{i}\right) & =\mathbf{p}(T, F)+\rho\left(\bigcap_{i=1}^{r} P K_{t}\right) \\
& =\mathbf{p}(T, F)+\rho\left(\sqrt{I_{f}}\right) \\
& =\mathbf{p}(T, F)+\rho \rho^{-1}\left(\sqrt{J_{f}}\right) \\
& =\sqrt{J_{f}} .
\end{aligned}
$$

REMARKS.

(1) The part of Corollary 1.13 which states that $\sqrt{J}_{f}=\bigcap_{i=1}^{r} \mathbf{p}\left(K_{\imath}, c_{\imath}\right)$, follows directly from Theorem 2.4 (ii), below.

(2) Let $\operatorname{Strat}\left(H_{T}(X)\right)=\{\mathbf{p}(K, c) \mid(K, c) \in \mathscr{T}(X)\}$, and let Strat $(R)=\{P K \mid K$ is a subtorus of $T\}$. Then Lemma 1.12 implies that the support functions enjoy the following commutativity: 


$$
\begin{aligned}
& \operatorname{Spec}\left(H_{T}(X)\right) \stackrel{\sigma}{\rightarrow} \operatorname{Strat}\left(H_{T}(X)\right) \\
& \rho^{*} \downarrow \quad \downarrow \rho^{*} \mid \operatorname{Strat}\left(H_{T}(X)\right) \\
& \operatorname{Spec}(R) \stackrel{\sigma}{\rightarrow} \operatorname{Strat}(R)
\end{aligned}
$$

(3) Since, with the notation of Lemma $1.10, \psi q=\bar{q} \rho, \psi$ being the isomorphism of Lemma 1.7, it follows easily that $K^{+} \rightarrow V(K, c)^{+} \subseteq$ $\operatorname{Spec}\left(H_{T}(X)\right)$, the map being $\mathbf{p} \rightarrow(K, c)^{*-1}(\mathbf{p})$.

We shall now prove Theorem 1.1 in the general situation, where $G$ is a compact connected Lie group, $T$ is a maximal torus of $G$, and $W$ is the Weyl group. The action of $W$ on $H_{T}(X)$ induces an action of $W$ on $\operatorname{Spec}\left(H_{T}(X)\right)$. The next lemma, together with Proposition 1.3 (ii), is the key to extending the results.

Lemma 1.14. Let $\mathbf{p} \in \operatorname{Spec}\left(H_{G}(X)\right)$. Let $\mathbf{a}=\mathbf{p} \cdot H_{T}(X)$ be the ideal of $H_{T}(X)$ generated by $\mathbf{p}$. Let $\mathbf{q} \in \operatorname{Spec}\left(H_{T}(X)\right)$ be such that $\mathbf{q} \cap H_{G}(X)=\mathbf{p}$. Then

$$
\sqrt{\mathbf{a}}=\bigcap_{w \in W} w \mathbf{q} .
$$

Proof. First, note that there always exists $\mathbf{q}$ such that $\mathbf{q} \cap H_{G}(X)=$ $\mathbf{p}$, by the Cohen-Seidenberg Theorem. Now $\mathbf{p} \subseteq \mathbf{q}$, and so $\mathbf{p}=w \cdot \mathbf{p} \subseteq$ $w \cdot \mathbf{q}$. Hence

$$
\mathbf{a} \subseteq \bigcap_{w \in W} w \cdot \mathbf{q}
$$

Suppose $x \in \bigcap_{w \in W} w \cdot \mathbf{q}$. Then, for any $w \in W, w x \in \mathbf{q}$. As before, consider $\prod_{w \in W}(x-w x)=0$. If the order of $W$ is $n$, then we obtain an equation $x^{n}+b_{1} x^{n-1}+\cdots+b_{n}=0$, where each $b_{\imath} \in \mathbf{q} \cap$ $H_{G}(X)$. Thus $x^{n} \in \mathbf{a}$.

COROLlaRY 1.15. There exists a homeomorphism

$$
f: \operatorname{Sepc}\left(H_{G}(X)\right) \stackrel{\sim}{\rightarrow} \operatorname{Spec}\left(H_{T}(X)\right) / W
$$

such that the diagram

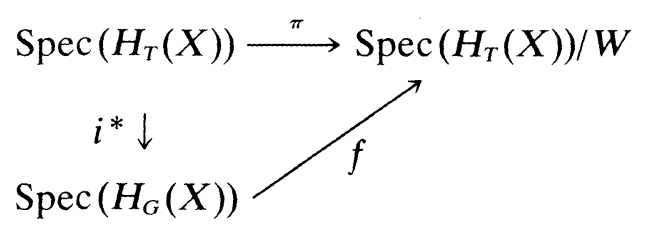

commutes, where $i^{*}$ is the restriction, and $\pi$ is the orbit map. 
Proof. Clearly there exists a well-defined continuous map $g: \operatorname{Spec}\left(H_{T}(X)\right) / W \rightarrow \operatorname{Spec}\left(H_{G}(X)\right)$, such that $g \pi=i^{*} ; g(W(\mathbf{q}))=$ $\mathbf{q} \cap H_{G}(X)$.

If $g(W(\mathbf{q}))=g\left(W\left(\mathbf{q}^{\prime}\right)\right)=\mathbf{p}$, say, then, with $\mathbf{p}, \mathbf{q}$ and $\mathbf{a}$ as in Lemma 1.14 , we have that $\mathbf{q}^{\prime} \supseteq \sqrt{\mathbf{a}}$, and hence $\mathbf{q}^{\prime} \supseteq w \mathbf{q}$, for some $w \in W$. Thuś $\mathbf{q}^{\prime}=w \mathbf{q}$, by the Cohen-Seidenberg Theorem, and hence $g$ is injective.

Finally, $i^{*}$ is closed and surjective by the Cohen-Seidenberg Theorem; and hence $g$ too is closed and surjective.

To distinguish between $H_{T}(X)$ and $H_{G}(X)$, we shall, for the remainder of this section, denote the elements of $\operatorname{Strat}\left(H_{T}(X)\right)$ by $\mathbf{q}(K, c)$, reserving the notation $\mathbf{p}(K, c)$ for $\operatorname{Spec}\left(H_{G}(X)\right)$.

LEMMA 1.16.

(i) $\mathbf{q}(K, c) \cap H_{G}(X)=\mathbf{p}(K, c)$

(ii) If $w \in W$ is represented by $g T$ in $N T / T$, then $w \cdot q(K, c)=$ $\mathbf{q}\left(g K g^{-1}, g c\right)$.

Proof. (i) is clear from the definition of $\mathbf{p}(K, c)$. To see (ii) consider the diagram

$$
\begin{array}{clrl}
B_{K} & \rightarrow c_{K} & \rightarrow X_{T} \\
\theta_{g} \downarrow & & \\
B_{g K g^{-1}} & \rightarrow(g c)_{g K g^{-1}} & \rightarrow \psi_{T}
\end{array}
$$

where for $z \in E_{G}\left(B_{G}=E_{G} / G\right), \theta_{g}$ is the map $K(z) \rightarrow g K g^{-1}(g z)$, and, for $x \in X, \psi_{g}$ is the map $T(x, z) \mapsto T(g x, g z) . \psi_{g}$ depends only on $w$, but $\theta_{g}$ depends upon the choice of $g$. Clearly the rows may be chosen so that the diagram commutes, and the composition on the top row gives $(K, c)^{*}$, while the composition on the bottom row gives $\left(\mathrm{gKg}^{-1}, g c\right)^{*}$, and clearly, the latter map depends only on $w$, since $t c=c$, for any $t \in T$. The result now follows since $\theta_{g}$ is a homeomorphism, and $\psi_{g}$ is the homeomorphism on $X_{T}$, which induces the action of $w^{-1}$ on $H_{T}(X)$.

Thus $\operatorname{Strat}\left(H_{T}(X)\right)$ is $W$-invariant, and we have the following lemma.

Lemma 1.17. $\sigma: \operatorname{Spec}\left(H_{T}(X)\right) \rightarrow \operatorname{Strat}\left(H_{T}(X)\right)$ is $W$-equivariant.

Proof. Let $\mathbf{q} \in \operatorname{Spec}\left(H_{T}(X)\right)$, let $\sigma(\mathbf{q})=\mathbf{q}(K, c)$, and let $w \in W$. Suppose that $w \mathbf{q} \supseteq \mathbf{q}(H, d)$ for some $(H, d) \in \mathscr{T}(X)$. Then $\mathbf{q} \supseteq w^{-1} \mathbf{q}(H, d)$, and so $\mathbf{q}(K, c) \supseteq w^{-1} \mathbf{q}(H, d)$. Thus $w \mathbf{q} \supseteq w \mathbf{q}(K, c) \supseteq$ $\mathbf{q}(H, d)$, and, hence $\sigma(w \mathbf{q})=w \sigma(\mathbf{q})=w \mathbf{q}(K, c)$.

We are now in a position to prove Theorem 1.1 as stated. 
Proof of Theorem 1.1. Given $\mathbf{p} \in \operatorname{Spec}\left(H_{G}(X)\right)$, choose $\mathbf{q} \in$ $\operatorname{Spec}\left(H_{T}(X)\right)$, such that $\mathbf{q} \cap H_{G}(X)=\mathbf{p}$, and set $\sigma(\mathbf{p})=\sigma(\mathbf{q}) \cap$ $H_{G}(X)$. By Lemma 1.17, $\sigma$ is a well-defined map of $\operatorname{Spec}\left(H_{G}(X)\right)$ onto Strat $\left(H_{G}(X)\right)=\left\{\mathbf{p}(K, c) \mid(K, c) \in I_{T}\right\}$, and $\sigma(\mathbf{p})$ is independent of the choice of q. Clearly, if $\mathbf{p} \supseteq \mathbf{p}(H, d)$, then $\sigma(\mathbf{p}) \supseteq \mathbf{p}(H, d)$, and so parts (i) and (iii) of the theorem are proved.

For $(K, c) \in I_{T}$, we have

$$
V(K, c)^{+}=\left\{\mathbf{p} \in \operatorname{Spec}\left(H_{G}(X)\right) \mid \sigma(\mathbf{p})=\mathbf{p}(K, c)\right\} .
$$

Let

$$
U(K, c)^{+}=\left\{\mathbf{q} \in \operatorname{Spec}\left(H_{T}(X)\right) \mid \sigma(\mathbf{q})=\mathbf{q}(K, c)\right\},
$$

and let $W_{0}=\{w \in W \mid w \mathbf{q}(K, c)=\mathbf{q}(K, c)\}$ be the isotropy subgroup of the $W$-action on $\operatorname{Spec}\left(H_{T}(X)\right)$ at $\mathbf{q}(K, c)$. Then $U(K, c)^{+}$is $W_{0^{-}}$ invariant by Lemma 1.17 .

As in Corollary 1.15, we have a homeomorphism

$$
U(K, c)^{+} / W_{0} \rightarrow V(K, c)^{+},
$$

defined by $W_{0}(\mathbf{q}) \rightarrow \mathbf{q} \cap H_{G}(X)$.

Now, representing $w \in W$ by $g T \in N T / T, g \in N T$, it follows from Lemma 1.16, that $w \in W_{0}$ if and only if $g \in \operatorname{Norm}(K, c)$. Thus $W_{0}$ is isomorphic to $(\operatorname{Norm}(K, c) \cap N T) / T$, which maps onto $W(K, c)$, with kernel $(Z K \cap N T) / T$.

Since $Z K$ is connected, it follows that $(Z K \cap N T) / T$ acts trivially on $U(K, c)^{+}$, and, hence, there is induced on $U(K, c)^{+}$an action of $W(K, c)$, with $U(K, c)^{+} / W(K, c)$ homeomorphic to $U(K, c)^{+} / W_{0}$.

$K^{+}$, however, is homeomorphic to $U(K, c)^{+}$; and so we have an induced $W(K, c)$-action on $K^{+}$, with $K^{+} / W(K, c)$ homeomorphic to $V(K, c)^{+}$, completing the proof of the theorem.

REMARK. The essence of the proof of Theorem 1.1 (i) and (iii) is that the homeomorphism, $f$, of Corollary 1.15 induces a homeomorphism $\bar{f}: \operatorname{Strat}\left(H_{G}(X)\right) \rightarrow \operatorname{Strat}\left(H_{T}(X)\right) / W$, such that $\bar{f} \sigma=\sigma^{\prime} f$, where $\sigma^{\prime}$ : $\operatorname{Spec}\left(H_{G}(X)\right) / W \rightarrow \operatorname{Strat}\left(H_{T}(X)\right) / W$ is induced from $\sigma: \operatorname{Spec}\left(H_{T}(X)\right)$ $\rightarrow \operatorname{Strat}\left(H_{T}(X)\right)$, by Lemma 1.17: and $\operatorname{Strat}\left(H_{T}(X)\right) / W$ is in one-to-one correspondence with $I_{T}$, by Lemma 1.16 (ii).

The support maps defined above are natural, in as much as we have the following theorem, whose proof is straightforward, and will be omitted. 


$$
\begin{array}{ccc}
\operatorname{Spec}(R) & \leftarrow \operatorname{Spec}\left(H_{T}(X)\right) \rightarrow \operatorname{Spec}\left(H_{G}(X)\right) \\
\sigma \downarrow & \sigma \downarrow & \sigma \downarrow \\
\operatorname{Strat}(R) & \leftarrow \operatorname{Strat}\left(H_{T}(X)\right) \rightarrow \operatorname{Strat}\left(H_{G}(X)\right)
\end{array}
$$

in which the horizontal maps are the obvious ones, is commutative and natural with respect to equivariant maps $(G, X) \rightarrow\left(G^{\prime}, X^{\prime}\right)$, where $G, G^{\prime}$ are compact connected Lie groups, $X, X^{\prime}$ satisfy conditions $(\mathrm{A})$ or $(B)$ and the maximal torus of $G^{\prime}$ is chosen to contain the image of the maximal torus of $G$.

REMARK. The support maps are not, in general, continuous with respect to Zariski topologies. The set of all $V(\mathbf{a})$, where $\mathbf{a}$ is an ideal in $H_{G}(X)$ such that $\mathbf{a}=(0)$ or $(1)$, or $\sqrt{\mathbf{a}}^{-} \bigcap_{t=1}^{r} \mathbf{p}\left(K_{t}, c_{t}\right)$, for any $\left(K_{t}, c_{\imath}\right) \in I_{T}$, is a topology of closed sets on $\operatorname{Spec}\left(H_{G}(X)\right)$; and if we take the subspace topology induced from this topology on $\operatorname{Strat}\left(H_{G}(X)\right)$, then, clearly, $\sigma$ is continuous.

2. The localization theorem and ideal theory. In [6], Chang and Skjelbred use the Localization Theorem of [7] (and [10]) to discuss the primary decomposition of certain ideals of geometric significance. This theory is concerned with $R$-module structures, and ideals in $R$. In this section, we shall recover similar results for $H_{T}(X)$ module structures and ideals in $H_{T}(X)$.

We shall be concerned only with torus actions on $X$, and we shall consider closed invariant subspaces of $X, Y$ and $Z$, which satisfy the conditions that $\operatorname{dim}_{0} H^{*}(Y)<\infty$, and $\operatorname{dim}_{0} H^{*}(Z)<\infty$. (Merely, we need $H_{T}^{*}(Y)$ and $H_{T}^{*}(Z)$ to be finitely generated $H_{T}(X)$-modules.) The long exact sequence of the pair $(X, Y)$ in equivariant cohomology is an exact sequence of $H_{T}(X)$-modules.

To simplify notation we shall denote the ring $H_{T}(X)$ by $B$. Recall, too, the notation of [10], $\S 3$, that $q: X \rightarrow X / T$ is the orbit projection, and if $u \in B$, then $\tilde{u}$ is the corresponding global section of the Leray sheaf on $X / T$. It is clear from the definition of $\tilde{u}$, that, in $B$,

$$
\mathbf{p}\left(T_{x}^{\circ}, F(x)\right)=\{u \in B \mid \tilde{u}(q(x))=0\},
$$

where $F(x)=F(T, x)$.

Definition. For $f \in B$, let $Y^{f}=\left\{x \in Y \mid f \notin \mathbf{p}\left(T_{x}^{\circ}, F(x)\right)\right\}$. Thus $Y^{f}=X \cap q^{-1}\{y \in X / T \mid \tilde{f}(y) \neq 0\}$; and so $Y^{f}$ is a closed invariant subspace of $X$. 
$H_{T}^{*}(Y) \rightarrow H_{T}^{*}\left(Y^{f}\right)$, induces a $B_{f}$-module isomorphism, $H_{T}^{*}(Y)_{f}$ $\stackrel{\sim}{\rightarrow} H_{T}^{*}\left(Y^{f}\right)_{f}$. (As usual, $B_{f}$ is the localization of $B$ with respect to the multiplicative set generated by $f$.)

Proof. Let $\varphi^{*}: H_{T}(X) \rightarrow H_{T}(Y)$ denote the restriction, and, for $y \in Y$, let

$$
\begin{gathered}
\mathbf{p}^{\prime}\left(T_{y}^{\circ}, F(y)\right)=\operatorname{ker}\left[H_{T}(Y) \rightarrow H_{T}(F(y)) \rightarrow H_{T_{y}^{\circ}}\right] \quad \text { Thus, } \\
\mathbf{p}^{\prime}\left(T_{y}^{\circ}, F(y)\right) \in \operatorname{Strat}\left(H_{T}(Y)\right), \quad \text { and } \mathbf{p}\left(T_{y}^{\circ}, F(y)\right)=\varphi^{*-1} \mathbf{p}^{\prime}\left(T_{y}^{\circ}, F(y)\right) .
\end{gathered}
$$

Suppose that $Y^{f}=\varnothing$. Then $\varphi^{*}(f) \in \mathbf{p}^{\prime}\left(T_{y}^{\circ}, F(y)\right)$, for all $y \in Y$. Hence $\varphi^{*}(f)$ is nilpotent in $H_{T}(Y)$, and we have $H_{T}^{*}(Y)_{f} \leftrightharpoons H_{T}^{*}\left(Y^{f}\right)_{f}=0$.

Now suppose that $Y^{f} \neq \varnothing$. Just as in [10], let $N$ be a closed invariant neighborhood of $Y^{f}$ in $Y$, and let $N^{\prime}=Y-\stackrel{\circ}{N}$. Then $\left(N^{\prime}\right)^{f}=$ $\varnothing$, and the Mayer-Vietoris sequence implies that $H_{T}^{*}(Y)_{f} \rightarrow H_{T}^{*}(N)_{f}$. Again, as in [10], the result follows from taking direct limits as $N$ varies.

We may now prove the Localization Theorem for $B$-module structures.

THEOREM 2.2. Let $\mathbf{p} \in \operatorname{Spec}(B)$, and suppose that $\sigma \mathbf{p}=$ $\mathbf{p}(K, c)$. Then, with respect to $B$-module structures, localization induces isomorphisms,

$$
H_{T}^{*}(Y)_{\mathbf{p}} \rightarrow H_{T}^{*}(c \cap Y)_{\mathbf{p}},
$$

and

$$
H_{T}^{*}(X, Y)_{\mathbf{p}} \rightarrow H_{T}^{*}(c, c \cap Y)_{\mathbf{p}}
$$

Proof. From the long exact sequence for the pair $(X, Y)$, the second isomorphism will follow from the first.

The first isomorphism follows from the continuity of sheaf cohomology, and Lemma 2.1, and the fact that

$$
c=\cap\left\{X^{f} \mid f \notin \mathbf{p}\right\} .
$$

REMARKS.

(1) Give Spec(B) the Zariski topology and let $D_{f}=$ $\{\mathbf{p} \in \operatorname{Spec}(B) \mid f \notin \mathbf{p}\}$ denote a typical basic open set. Let $\mathscr{F}$ denote the sheaf, $D_{f} \rightarrow H_{T}^{*}(Y)_{f}$, and let $\mathscr{G}$ denote the presheaf, $D_{f} \rightarrow$ $H_{T}^{*}\left(Y^{f}\right)_{f}$. Then Lemma 2.1 says that $\mathscr{F}$ and $\mathscr{G}$ are isomorphic as presheaves, and hence, as sheaves. The first isomorphism of Theorem 2.2 is then the isomorphism induced on the stalks at $\mathbf{p .}$

(2) Given $\mathbf{p}(K, c)$, let $\mathbf{r}(K, c)=\operatorname{ker}\left[H_{T}(c) \rightarrow H_{K}\right]$. Then it is easy to show that restriction induces a ring isomorphism, $H_{T}(X)_{\mathrm{p}(K, c)}$ $\stackrel{\rightarrow}{\rightarrow} H_{T}(c)_{\mathbf{r}(K, c)}$. 
We have the following analogue of [6], Lemma 1.1.

Proposition 2.3. Let $\mathbf{p} \in \operatorname{Spec}(B)$, and suppose that $\sigma \mathbf{p}=$ $\mathbf{p}(K, c)$. Let $N$ be the $B$-module $H_{T}^{*}(c, c \cap Y) \bigotimes_{H_{T}(c)} H_{T}^{*}(c \cap Z)$. Then the localization map, $N \rightarrow N_{\mathrm{p}}$, is a monomorphism.

Proof. Let $L=T / K$, so that $H_{T}^{*}(c, c \cap Y)=H_{K} \otimes H_{L}^{*}(c, c \cap Y)$, $H_{T}^{*}(c \cap Z)=H_{K} \otimes H_{L}^{*}(c \cap Z)$, and $H_{T}(c)=H_{K} \otimes H_{L}(c)$. Then the middle four interchange ([9]), gives an isomorphism

$H_{T}^{*}(c, c \cap Y) \otimes_{H_{T(c)}} H_{T}^{*}(c \cap Z) \rightarrow H_{K} \otimes\left[H_{L}^{*}(c, c \cap Y) \otimes_{H_{L}(c)} H_{L}^{*}(c \cap Z)\right]$.

The result now follows just as in [6].

Let $M$ be a $B$-submodule of $H_{T}^{*}(X, Y) \otimes_{B} H_{T}^{*}(Z)$. For a given $(K, c) \in \mathscr{T}(X)$, denote by $M(K, c)$, the image of $M$ under restriction into $H_{T}^{*}(c, c \cap Y) \bigotimes_{H_{T}(c)} H_{T}^{*}(c \cap Z)$.

Definition. With the above notation, let ann $(M)$ be the annihilator of $M$ in $B$, and let $\operatorname{ann}^{(K, c)}(M)$ be the annihilator of $M(K, c)$ in $B$. We shall say that a pair $(K, c) \in \mathscr{T}(X)$ belongs to $M$ if and only if

(i) $\quad \operatorname{ann}^{(K, c)}(M) \neq(1)$; and

(ii) if $(H, d)>(K, c)$, (i.e. $(H, d) \geqq(K, c)$ and $(H, d) \neq(K, c))$, then $\operatorname{ann}^{(H, d)}(M)=(1)$.

The following results may be proved in a manner strictly analogous to the corresponding results of [6], using Theorem 2.2 and Proposition 2.3 .

THEOREM 2.4 .

(i) if $\mathbf{p} \in \operatorname{Spec}(B)$, and $\sigma \mathbf{p}=\mathbf{p}(K, c)$, then $\operatorname{ann}(M)_{\mathbf{p}} \cap B=$ $\operatorname{ann}\left(M_{\mathrm{p}}\right) \cap B=\operatorname{ann}^{(K, c)}(M)$.

(ii) $\sqrt{\operatorname{ann}(M)}=\cap\{\mathbf{p}(K, c) \mid(K, c)$ belongs to $M\}$.

COROllary 2.5 .

(i) If $(K, c)$ belongs to $M$, then $\operatorname{ann}^{(K, c)}(M)$ is primary, with $\sqrt{\operatorname{ann}^{(K, c)}(M)}=\mathbf{p}(K, c)$.

(ii) If $(K, c)$ belongs to $M$, then

$$
\operatorname{ann}^{(K, c)}(M)=\{x \in B \mid \operatorname{ann}(M):(x) \not \subset \mathbf{p}(K, c)\} .
$$

THEOREM 2.6. There exists a reduced primary decomposition of $\operatorname{ann}(M)$ of the form $\operatorname{ann}(M)=\cap\left\{\operatorname{ann}^{(K, c)}(M) \mid(K, c)\right.$ belongs to $\left.M\right\}$ $\cap \mathbf{q}_{1} \cap \cdots \cap \mathbf{q}_{m}$, where, for each $i, 1 \leqq i \leqq m, \sqrt{\mathbf{q}_{i}}=\mathbf{p}\left(H_{i}, d_{i}\right)$, for some $\left(H_{i}, d_{i}\right) \in \mathscr{T}(X)$, which does not belong to $M$, but for which $(K, c) \geqq\left(H_{i}, d_{i}\right)$ for some $(K, c)$, which does belong to $M$; and 


$$
\operatorname{ann}^{\left(H_{i} d_{i}\right)}(M) \subset \cap\left\{\operatorname{ann}^{(K, c)}(M) \mid(K, c)>\left(H_{i}, d_{l}\right)\right\}
$$

Remark. Let $F$ be a component of $X^{T}$, assuming that $X^{T} \neq \varnothing$. Let $M=H_{T}^{*}\left(X, X^{T}\right) \otimes_{B} H_{T}^{*}(F)$, and let $N=H_{T}^{*}(X, F) \otimes_{B} H_{T}^{*}(F)$. It is easily verified that $\operatorname{Supp}(M)=\operatorname{Supp}(N)$; and indeed, from [3], for example, we have that

$$
\begin{aligned}
\operatorname{Supp}(M) & =V(\operatorname{ann}(M))=\operatorname{Supp}\left(H_{T}^{*}\left(X, X^{T}\right)\right) \cap \operatorname{Supp}\left(H_{T}^{*}(F)\right) \\
& =\operatorname{Supp}\left(H_{T}^{*}(X, F)\right) \cap \operatorname{Supp}\left(H_{T}^{*}(F)\right) \\
& =\operatorname{Supp}(N)=V(\operatorname{ann}(N)) .
\end{aligned}
$$

Thus, if $X$ is a Poincare duality space over $Q$, the local weights at $F$, and their corresponding $F$-varieties ([1]), are precisely the pairs, $(K, c)$, which belong to $M$, or, equivalently, which belong to $N$.

3. An Application. We shall say that an action of a torus, $T$, on a space, $X$, is toricly uniform, if given any two subtori, $H$ and $K$, such that $H \subseteq K$, and $X^{K} \neq \varnothing$, then every component of $X^{H}$ contains at least one component of $X^{K}$; that is $c \cap X^{K} \neq \varnothing$, for every component, $c$, of $X^{H}$. A space, $X$, will be called toricly uniform, if every torus action on $X$ is toricly uniform. It is clear that $X$ is toricly uniform if, and only if, every torus action on $X$ with a nonempty fixed point set is toricly uniform.

Toricly uniform spaces are common. For example, if the even rational homotopy groups of $X$ are zero, then $X$ is toricly uniform, since all nonempty fixed point sets are connected in this case ([4]). It will follow from Corollary 3.4 below, that $X$ is toricly uniform if $H^{\text {odd }}(X)=$ 0 . Thus, if $X$ has the rational cohomology of complex or quaternionic projective space, then $X$ is toricly uniform. The following is a simple example of a nontoricly uniform action.

ExAmple. Let $Y=S^{2} \times S^{1}$, and let the torus $T^{2}=S^{1} \times S^{1}$ act on $Y$ by the product action of $S^{1}$ rotating $S^{2}$ about an axis through the north and south poles, $n$ and $s$, and $S^{1}$ acting on $S^{1}$ by multiplication. Let $A$ be the invariant subspace $\{n\} \times S^{1}$, and let $X$ be the quotient space $Y / A$. Clearly $X$ inherits a $T^{2}$-action from $Y$; and this action is not toricly uniform, since $T^{2}$ fixes the single point $A / A$, and the first factor of $T^{2}$ fixes this point plus $\{s\} \times S^{1}$.

We shall use the ideals, $\mathbf{p}(K, c)$, to give the following algebraic characterization of toricly uniform actions. In $H_{T}(X)$, let $N$ be the ideal of $R$-torsional elements.

THEOREM 3.1. If the torus $T$ acts on $X$, such that $X^{T} \neq \varnothing$, then the action is toricly uniform, if, and only if, $N \subseteq \sqrt{(0)}$ in $H_{T}(X)$. 
REMARK. If $X^{T}$ is not necessarily nonempty, then the action is toricly uniform if and only if $M \subseteq \sqrt{(0)}$, where the ideal $M$ is the intersection of all $\operatorname{ker}\left[H_{T}(X) \rightarrow H_{T}\left(X^{K}\right)\right], K$ ranging over the maximal subtori of $T$ with $X^{K} \neq \varnothing$. The proof is strictly analogous to the proof of Theorem 3.1 as stated. By [7], $M$ may be characterized algebraicly as the set of all $x \in H_{T}(X)$, such that $\operatorname{ann}(x)$ and $J=\operatorname{ker}\left[\rho: R \rightarrow H_{T}(X)\right]$ have no common minimal primes.

For the proof of Theorem 3.1 we need the following proposition.

Proposition 3.2. If a torus $T$ acts on $X$ such that $X^{T} \neq \varnothing$, and if $F^{\prime}$, $1 \leqq i \leqq s$, are the components of $X^{T}$, then

$$
\sqrt{N}=\bigcap_{i=1}^{s} \mathbf{p}\left(T, F^{i}\right) .
$$

Proof. Let $\varphi:\left(X^{T}\right)_{T} \rightarrow X_{T}, \varphi_{1}: F_{T}^{i} \rightarrow X_{T}, 1 \leqq i \leqq s$, be the inclusions. The localization theorem of [7] or [10] implies that $N=\operatorname{ker} \varphi^{*}$. Clearly $\operatorname{ker} \varphi^{*}=\bigcap_{l=1}^{s} \operatorname{ker} \varphi_{\imath}^{*}$.

Now $\mathbf{p}\left(T, F^{i}\right)=\varphi_{1}^{*-1}\left(R \otimes \tilde{H}\left(F^{i}\right)\right)$, and so $\sqrt{\operatorname{ker} \varphi_{i}^{*}}=\mathbf{p}\left(T, F^{i}\right)$. The result follows.

COROLlary 3.3 ([7]). $\quad X^{T}$ is connected if, and only if, $\sqrt{N}$ is prime.

Proof of Theorem 3.1. Clearly the action is toricly uniform if, and only if, $\bigcap_{t=1}^{s} \mathbf{p}\left(T, F^{i}\right)=\sqrt{(0)}$. The theorem follows.

The following corollary also follows easily from the localization theorem.

Corollary 3.4. If the torus $T$ acts on $X$ such that $X$ is totally nonhomologous to zero (with respect to rational cohomology) in $X_{T} \rightarrow B_{T}$, then the action is toricly uniform.

Proof. $\quad N=(0)$ in this case.

Concluding Remarks. The ideals, $\mathbf{p}(K, c)$, or similar ideals in a closely analogous context were used to great effect, with varying degrees of explicitness, by Hsiang, Chang and Skjelbred in [7], [6] and [13]. Indeed, one could rework the program of [7] in the following way. Let $\pi: R\left[x_{1}, \cdots, x_{k}\right] \rightarrow H_{T}(X) / \sqrt{(0)}$ be an $R$-algebra epimorphism; suppose that $X^{T} \neq \varnothing$, and let $F^{\prime}, 1 \leqq i \leqq s$, be the components of $X^{T}$; let $\alpha_{j}^{i}=\left(T, F^{i}\right)^{*} \pi\left(x_{j}\right) \in R$. Then $\pi^{-1} \mathbf{p}\left(T, F^{i}\right)=\left(x_{1}-\alpha_{1}^{i}, \cdots, x_{k}-\alpha_{k}^{i}\right)$ for $1 \leqq i \leqq s$; and we have that

$$
\begin{aligned}
{\left[\pi^{-1} \mathbf{p}\left(T, F^{\prime}\right)+\pi^{-1} \mathbf{p}\left(T, F^{\prime}\right)\right] \cap R } & =\rho^{-1}\left[\mathbf{p}\left(T, F^{i}\right)+\mathbf{p}\left(T, F^{\prime}\right)\right] \\
& =\left(\alpha_{1}^{i}-\alpha_{1}^{\prime}, \cdots, \alpha_{k}^{\prime}-\alpha_{k}^{\prime}\right) .
\end{aligned}
$$


The latter ideal has, therefore, minimal primes of the form $P K$ in $R$; and it is a very useful ideal for describing the geometry of connecting subtori, as was done in [7], [6] and [13]. (Cf. Corollary 1.9, above).

\section{REFERENCES}

1. C. J. Allday and T. Skjelbred, The Borel formula and the topological splitting principle for torus actions on a Poincaré duality space, Ann. of Math., 100 (1974), 322-325.

2. C. J. Allday, Torus actions on a cohomology product of three odd spheres, Trans. Amer. Math. Soc., 203 (1975), 343-358.

3. N. Bourbaki, Algèbre Commutative, Hermann, Paris (1961).

4. G. E. Bredon, Homotopical properties of fixed point sets of circle group actions, I., Amer. J. Math., 91 (1969), 874-888.

5. _ Introduction to Compact Transformation Groups, Academic Press, New York (1972).

6. T. Chang and T. Skjelbred, The topological Schur lemma and related results, Ann. of Math., 100 (1974), 307-321.

7. W.-Y. Hsiang, On some fundamental theorems in cohomology theory of topological transformation groups, Taita J. Math., 2 (1970), 61-87.

A summary of these results may be found in:

W.-Y. Hsiang, Some fundamental theorems in cohomology theory of topological transformation groups, Bull. Amer. Math. Soc., 77 (1971), 1094-1098.

8. W.-Y. Hsiang and T. Skjelbred, Untitled lecture notes, in preparation.

9. S. MacLane, Homology, Springer-Verlag, Berlin (1963).

10. D. Quillen, The spectrum of an equivariant cohomology ring: I, Ann. of Math., 94 (1971), 549-572.

11. D. Quillen, The spectrum of an equivariant cohomology ring: II, Ann. of Math., 94 (1971), 573-602.

12. J.-P. Serre, Algèbre Locale, Multiplicités, Lecture Notes in Mathematics No. 11, Springer-Verlag, Berlin (1965).

13. T. Skjelbred, Thesis, University of California, Berkeley (1972).

Received July 17, 1974.

UNIVERSITY OF HAWAII 



\section{PACIFIC JOURNAL OF MATHEMATICS}

\section{EDITORS}

RICHARD ARENS (Managing Editor)

University of California

Los Angeles, California 90024

\section{J. Dugundu}

Department of Mathematics University of Southern California Los Angeles, California 90007

D. Gilbarg and J. Milgram Stanford University Stanford, California 94305

\section{ASSOCIATE EDITORS}
E. F. BECKENBACH
B. H. NeumanN
F. WOLF
K. YoSHIDA

\section{SUPPORTING INSTITUTIONS}

UNIVERSITY OF BRITISH COLUMBIA CALIFORNIA INSTITUTE OF TECHNOLOGY

UNIVERSITY OF CALIFORNIA

MONTANA STATE UNIVERSITY

UNIVERSITY OF NEVADA

NEW MEXICO STATE UNIVERSITY

OREGON STATE UNIVERSITY

UNIVERSITY OF OREGON

OSAKA UNIVERSITY

\author{
UNIVERSITY OF SOUTHERN CALIFORNIA \\ STANFORD UNIVERSITY \\ UNIVERSITY OF HAWAII \\ UNIVERSITY OF TOKYO \\ UNIVERSITY OF UTAH \\ WASHINGTON STATE UNIVERSITY \\ UNIVERSITY OF WASHINGTON \\ AMERICAN MATHEMATICAL SOCIETY
}

The Supporting Institutions listed above contribute to the cost of publication of this Journal, but they are not owners or publishers and have no responsibility for its contents or policies.

Mathematical papers intended for publication in the Pacific Journal of Mathematics should be in typed form or offset-reproduced (not dittoed), double spaced with large margins. Underline Greek letters in red, German in green, and script in blue. The first paragraph or two must be capable of being used separately as a synopsis of the entire paper. Items of the bibliography should not be cited there unless absolutely necessary, in which case they must be identified by author and Journal, rather than by item number. Manuscripts, in duplicate, may be sent to any one of the four editors. Please classify according to the scheme of Math. Reviews, Index to Vol. 39. All other communications should be addressed to the managing editor, or Elaine Barth, University of California, Los Angeles, California, 90024.

100 reprints are provided free for each article, only if page charges have been substantially paid. Additional copies may be obtained at cost in multiples of 50 .

The Pacific Journal of Mathematics is issued monthly as of January 1966. Regular subscription rate: $\$ 72.00$ a year (6 Vols., 12 issues). Special rate: $\$ 36.00$ a year to individual members of supporting institutions.

Subscriptions, orders for back numbers, and changes of address should be sent to Pacific Journal of Mathematics, 103 Highland Boulevard, Berkeley, California, 94708.

PUBLISHED BY PACIFIC JOURNAL OF MATHEMATICS, A NON-PROFIT CORPORATION

Printed at Jerusalem Academic Press, POB 2390, Jerusalem, Israel.

$$
\begin{gathered}
\text { Copyright } 1976 \text { Pacific Journal of Mathematics } \\
\text { All Rights Reserved }
\end{gathered}
$$




\section{Pacific Journal of Mathematics}

Vol. 62, No. 2

February, 1976

Allan Russell Adler and Catarina Isabel Kiefe, Pseudofinite fields, procyclic

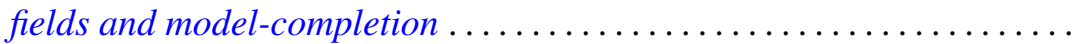

Christopher Allday, The stratification of compact connected Lie group

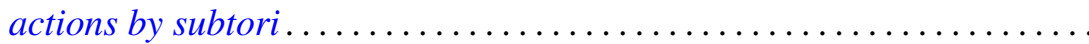

Martin Bartelt, Commutants of multipliers and translation operators .......

Herbert Stanley Bear, Jr., Ordered Gleason parts ..................

James Robert Boone, On irreducible spaces. II .....................

James Robert Boone, On the cardinality relationships between discrete

collections and open covers ............................

L. S. Dube, On finite Hankel transformation of generalized functions .......

Michael Freedman, Uniqueness theorems for taut submanifolds . . . . . . . . .

Shmuel Friedland and Raphael Loewy, Subspaces of symmetric matrices

containing matrices with a multiple first eigenvalue .............

Theodore William Gamelin, Uniform algebras spanned by Hartogs

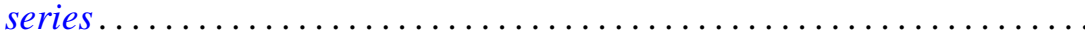

James Guyker, On partial isometries with no isometric part ............

Shigeru Hasegawa and Ryōtarō Satō, A general ratio ergodic theorem for

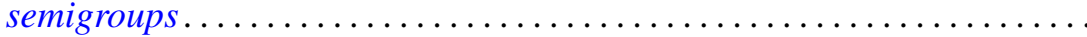

Nigel Kalton and G. V. Wood, Homomorphisms of group algebras with norm less than $\sqrt{2}$.

Thomas Laffey, On the structure of algebraic algebras...

Will Y. K. Lee, On a correctness class of the Bessel type differential operator $S_{\mu}$

Robert D. Little, Complex vector fields and divisible Chern classes ....

Kenneth Louden, Maximal quotient rings of ring extensions . .

Dieter Lutz, Scalar spectral operators, ordered $l^{\rho}$-direct sums, and the

counterexample of Kakutani-McCarthy . .

Ralph Tyrrell Rockafellar and Roger Jean-Baptiste Robert Wets, Stochastic

convex programming: singular multipliers and extended duality

singular multipliers and duality.

Edward Barry Saff and Richard Steven Varga, Geometric overconvergence of rational functions in unbounded domains ..........

Joel Linn Schiff, Isomorphisms between harmonic and P-harmonic Hardy

spaces on Riemann surfaces.

Virinda Mohan Sehgal and S. P. Singh, On a fixed point theorem of

Krasnoselskii for locally convex spaces.

Lewis Shilane, Filtered spaces admitting spectral sequence operations

Michel Smith, Generating large indecomposable continua . 